\title{
New Surgical Drapes for Observation of the Lower Extremities during Abdominal Aortic Repair
}

\author{
Yukio Obitsu, MD, Ph.D, Hiroshi Shigematsu, MD, Ph.D, Kazuhiro Satou, MD, Yoshiko Watanabe, MD, Ph.D, \\ Naozumi Saiki, MD, and Nobusato Koizumii, MD, Ph.D
}

\begin{abstract}
Objective: For the early diagnosis and therapy of peripheral thromboembolism (TE) as a complication of abdominal aortic repair (AAR), we developed and evaluated the usefulness of surgical drapes that permit observation of the lower extremities during AAR.
\end{abstract}

Materials and Methods: Between January 2007 and June 2009, the handling, durability, and usefulness of new surgical drapes were evaluated during AAR in 157 patients with abdominal aortic aneurysms and 9 patients with peripheral arterial disease. The drapes are manufactured by Hogy Medical Co. Ltd. and made of a water-repellent, spun lace, non-woven fabric, including a transparent polyethylene film that covers the patients' legs. This transparent film enables inspection and palpation of the lower extremities during surgery for early diagnosis and therapy of peripheral TE.

Results: As a peripheral complication, 1 patient had right lower extremity TE. This was diagnosed immediately after anastomosis, thrombectomy was performed, and the remaining clinical course was uneventful. In all patients, the drapes permitted observation of the lower extremities, and the dorsal arteries were palpable. There were no problems with durability.

Conclusions: New surgical drapes permit observation of the lower extremities during AAR for early diagnosis and treatment of peripheral TE.

Key words: abdominal aortic aneurysms, peripheral artery disease, surgical drapes

\section{INTRODUCTION}

$\mathrm{C}$ omplications affecting the prognosis after vascular reconstructive surgery in patients with abdominal aortic aneurysms (AAA) and peripheral arterial disease (PAD) include ischemic heart disease, cerebrovascular disease, hemorrhage, respiratory distress, gastrointestinal ischemia, and acute lower limb ischemia, and the last of

Department of Vascular Surgery, Tokyo Medical University, Tokyo, Japan

Received: March 232010 Accepted: July 20, 2010

Address for reprint requests to Yukio Obitsu, MD, Ph.D: Department of Vascular Surgery, Tokyo Medical University, 6-7-1

Nishishinjuku, Shinjuku-ku, Tokyo, 160-8402, Japan

Tel: +81-3-3342-6111, Fax: +81-3-3342-6193

E-mail: obitsu@tokyo-med.ac.jp these is an important complication of abdominal aortic surgery. Peripheral thromboembolism (TE) may be caused by the release of atheromatous debris or cholesterin crystals from the aortic wall, thrombosis of vascular grafts, or peripheral artery thrombosis. Important factors in preventing peripheral TE include selection of the clamping site, shortening of clamping time, careful anastomosis of the aorta, management of vascular grafts, and systemic heparinization. However, if peripheral TE does develop, any difficulty in observing the lower limbs during abdominal aortic repair may delay the diagnosis, leading to serious complications, including major amputation or other life-threatening events. Therefore, early diagnosis allowing prompt treatment is essential. This includes intraoperative monitoring, such as transcutaneous oxygen tension measurement and Doppler ultrasound.

For the early diagnosis and therapy of peripheral TE 


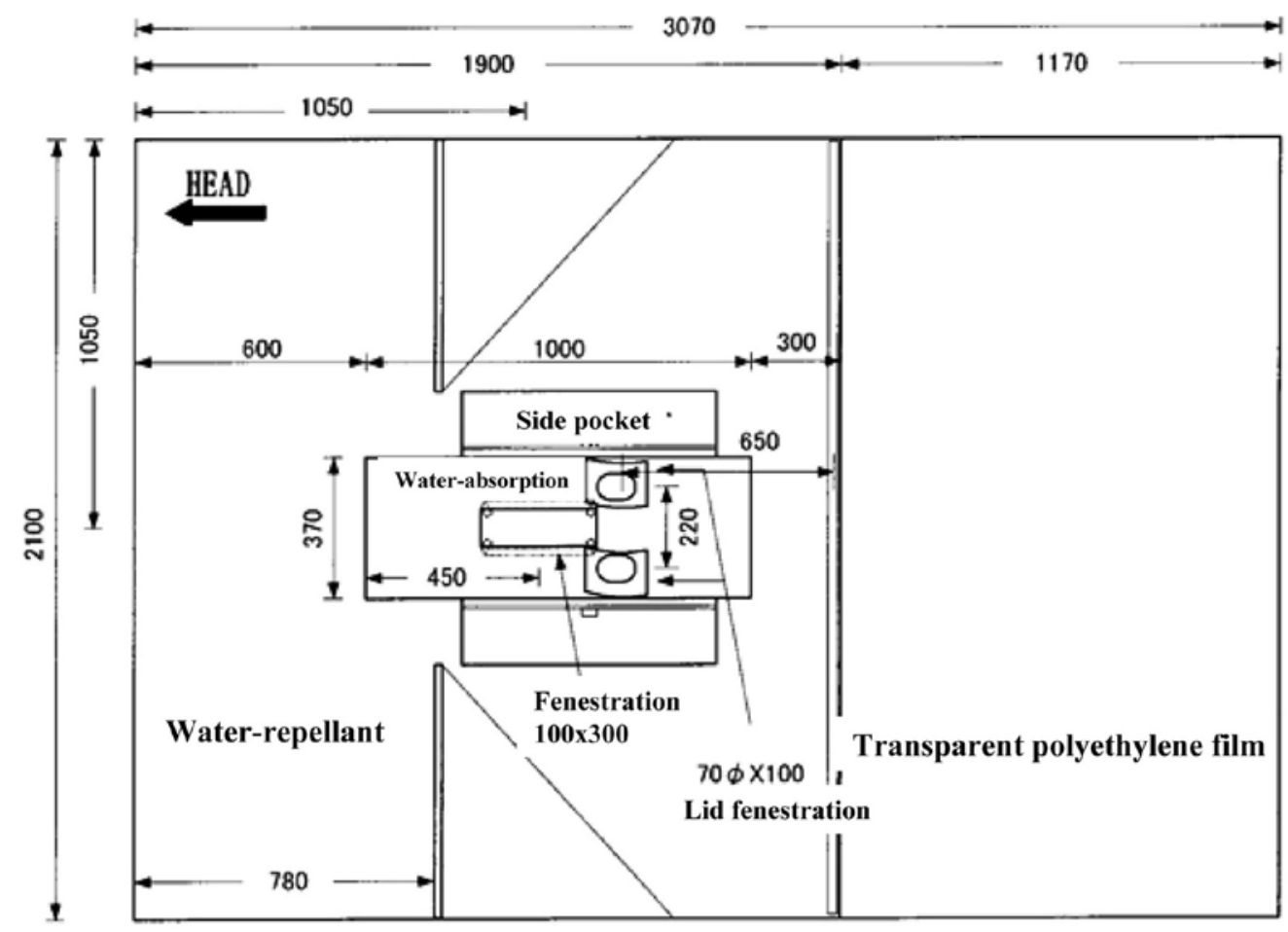

Fig. 1 Drawing of the transparent surgical drape.

during abdominal aortic repair to avoid complications such as "trash foot", we developed and evaluated the usefulness of a partly transparent surgical drape that permits inspection and palpation of the lower extremities during surgery.

\section{Materials ANd Methods}

The handling, durability, and usefulness of the surgical drapes were retrospectively evaluated in 166 consecutive patients $(\mathrm{M}: \mathrm{F}=147: 19$; age, $73.9 \pm 7.4$ years $)$ who underwent abdominal aortic repair for vascular graft reconstruction at our institution between January 2007 and June 2009. The drapes are manufactured by Hogy Medical Co. Ltd. and made of a water-repellent, spun lace, non-woven fabric. The drapes' special features include: 1) a window for laparotomy and covered windows bilaterally in the inguinal region for femoral artery procedures; 2) polyethylene film side pockets; and 3) transparent polyethylene film in the area that covers the patient's legs. This transparent film enables inspection and palpation of the lower extremities during surgery for early diagnosis and treatment of peripheral complications such as "trash foot" (Figs. 1 and 2).
Of the 166 patients, elective surgery was performed for AAA in 129, for iliac artery aneurysm in 16, and for PAD in 9. Urgent surgery was performed in 12 patients (rupture, 9; aneurysm-duodenal fistula, 3). In addition, 3 patients who had elective surgery for AAA had concurrent PAD, and 3 had blue toe syndrome with a shaggy aorta. Surgery involved laparotomy in 159 patients and a retroperitoneal approach in 7 patients. Except in cases of rupture or shaggy aorta, heparin was administered to maintain an activated clotting time for 200 seconds or more, and the aorta was clamped. Suprarenal aortic clamping was required in 16 patients, and renal artery reconstruction was performed in 3. After proximal anastomosis, blood was carefully flushed out, and to prevent release of debris distally, backflow was confirmed before distal anastomosis. A bifurcated graft was used in 132 patients, and a straight graft in 33. In 1 patient with aneurysm-duodenal fistula, the aorta was transected, and an axillo-bifemoral bypass was performed. Through an inguinal window in the drape, distal anastomosis to the common femoral artery was performed in 11 patients.

Use of the surgical drapes was approved by the Ethics Committee of Tokyo Medical University. 


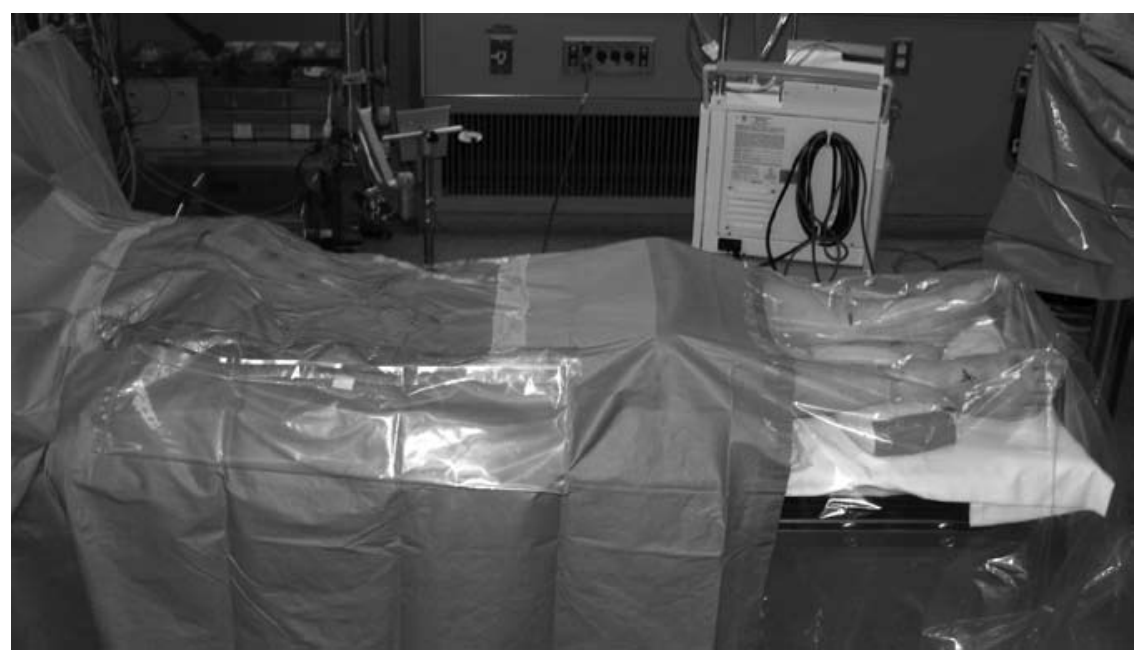

Fig. 2 Whole image of the transparent surgical drape.

\section{RESULTS}

Of the 154 elective surgery patients, 2 died as a result of aspiration pneumonia, and 1 of a perioperative acute type A aortic dissection (in-hospital mortality rate: $1.9 \%$ ). The remaining patients experienced uneventful postoperative courses, with no serious complications or sequelae, and all of them were ambulatory when they were discharged from the hospital. Of the 12 urgent surgery patients, 1 underwent a cardiac arrest during anesthesia induction, thoracic aortic clamping, and died; 1 with aortic aneurysm-duodenal fistula also died with multiple organ failure (in-hospital mortality rate: 16.7\%). One patient was transferred for rehabilitation. The other 9 patients were ambulatory and discharged from the hospital.

As for peripheral complications, in 1 patient $(0.6 \%)$, immediately after straight graft replacement with bilateral common iliac artery clamping, right superficial femoral artery TE was diagnosed early by inspection and palpation of the lower extremities. Thrombectomy was performed through the inguinal window of the drape, and the patient's remaining clinical course was uneventful. In 3 patients with AAA and shaggy aorta, in whom blue toe syndrome was a clear possibility, there was no worsening of the peripheral lesions, and the clinical course was satisfactory. However, 1 patient later underwent toe amputation for infection of the necrotic toe tip. In the 12 patients with surgical incisions in the inguinal region, including the 1 patient with intraoperative thrombectomy, removing the cover of the drape window facilitated surgical access to the femoral artery. In all patients, the drapes permitted observation of the lower extremities during surgery, and the dorsal arteries were palpable. The durability of the drapes was good, and no tears or other damage occurred.

\section{Discussion}

Peripheral thromboembolism, a serious complication of abdominal aortic surgery, may be caused by the release of atheromatous debris or cholesterin crystals from the aortic wall (e.g., during aortic clamping or clamp release), by thrombosis of vascular grafts, or by peripheral artery thrombosis. The incidence of peripheral TE in abdominal aortic surgery is $0.3 \%$ to $2.0 \% .^{1-3)}$ This incidence is not particularly high, but once peripheral TE occurs, serious or life-threatening consequences may develop.

Galland reported acute lower limb ischemia in $6.4 \%$ of patients who died after elective abdominal aortic surgery .) In a study of 1601 patients who underwent vascular reconstruction of the abdominal aorta, Kuhan et al. reported trash foot in 44 (2.0\%) limbs of 32 patients. Of these patients, 9 (28.1\%) underwent major amputations, minor amputations in 6 patients (18.6\%), and 8 (25\%) died within 30 days. ${ }^{2)}$ The odds ratio for death in patients with major amputations is 4.09 (CI, 2.78-6.0). ${ }^{3)}$ Moreover, in patients with a prior history of peripheral TE (e.g., blue toe syndrome) who undergo abdominal aortic repair, the incidence rates of amputations, renal failure, and death are all significantly higher than in those with no such history., 5)

Peripheral TE can lead to serious consequences and requires prevention. Preventive measures include adequate heparinization, clamping where the aortic wall is healthy, shortening of clamp times, flushing of aortic anastomosis sites, flushing of vascular grafts during anas- 
tomosis, and flushing out of blood after anastomosis to remove debris. In patients with AAA and a shaggy aorta, systemic heparinization is contraindicated. ${ }^{6)}$ In these cases, anticoagulation therapy with nafamostat mesilate or local heparinization is necessary. CT imaging is useful in the diagnosis of shaggy aorta and is important in the preoperative evaluation of all patients with AAA.)

If peripheral TE does develop, early diagnosis and prompt treatment are essential; and intraoperative monitoring of lower extremity hemodynamics, including transcutaneous oxygen tension measurement and Doppler ultrasound for high intensity transient signals (HITS), can be useful. ${ }^{7,8)}$ However, monitoring is unable to detect toe lesions (e.g., trash foot). Detection is also less effective in patients who also have PAD. Furthermore, monitoring is cumbersome and can be expensive.

On the other hand, the use of transparent drapes, although not providing quantitative evaluation, does permit visual inspection of the lower extremities during surgery, and the dorsal and posterior tibial artery pulses can be easily palpated. In addition, these drapes are convenient and inexpensive. In our study, 1 patient $(0.6 \%)$ developed peripheral TE. However, use of the transparent drapes facilitated early diagnosis, prompt treatment by thrombectomy, and a good vascular reconstruction outcome. In patients with blue toe syndrome and a shaggy aorta, the transparent drapes also permitted frequent observation of the lower limbs during aortic clamping and monitoring of anticoagulation therapy. It is difficult to distinguish the TE from peripheral circulatory collapse especially in patients with ruptured AAA, we can surmise to compare with inspections of before and after the reconstruction.

In endovascular aortic repair (EVAR), acute lower limb ischemia occurs as a complication due to atheromatous debris or cholesterin crystals from the aortic wall, technical difficulties during femoral artery clamping, or peripheral artery thrombosis. The incidence may be higher than with open repair., ${ }^{90)}$ Our study did not include patients who underwent EVAR. However, for peripheral TE as a complication of EVAR, as with open repair, early diagnosis and prompt treatment are essential. Thus, observation of the lower extremities can also provide effective monitoring during EVAR. The transparent surgical drapes may also be promising as "EVAR drapes".

\section{Conclusion}

Since delayed diagnosis of peripheral TE during abdominal aortic repair can have serious consequences, early diagnosis and prompt treatment are essential. The use of transparent drapes permits inspection and palpation of the lower extremities during surgery. This makes possible early diagnosis and treatment of peripheral TE. Like love, in the words of William Shakespeare, "It adds a precious seeing to the eye".

\section{ACKNOWLEDGEMENT}

The authors are indebted to Mr. C. W. P. Reynolds of the Department of International Medical Communications of Tokyo Medical University for his careful review of this manuscript.

\section{REFERENCES}

1) Galland RB, Wolfe JH. Mortality following elective infrarenal aortic reconstruction: a Joint Vascular Research Group study. Br J Surg 1998; 85: 633-6.

2) Kuhan G, Raptis S. Trash foot following operations involving the abdominal aorta. Aust N Z J Surg. 1997; 67: $21-4$.

3) Eliason JL, Wainess RM, Dimick JB, Cowan JA Jr, Henke PK, Stanley JC, et al. The effect of secondary operation on mortality following abdominal aortic aneurysm repair in the United States: 1988-2001. Vasc Endovascular Surg. 2005; 39: 465-72.

4) Baxter BT, McGee GS, Flinn WR, McCarthy WJ, Pearce WH, Yao JS. Distal embolization as a presenting symtom of aortic aneurysms. Am J Surg. 1990; 160: 197-201.

5) Sharma PV, Babu SC, Shah PM, Nassoura ZE. Changing patterns of atheroembolism. Cardovasc Surg. 1996; 4: 573-9.

6) Fine MJ, Kapoor W, Falanga V. Cholesterol crystal embolization: a review of 221 cases in the English literature. Angiology. 1987; 38: 769-84.

7) Kudo T, Inoue Y, Sugano N, Iwai T. Doppler ultrasound monitoring for detection of microembolic signals in peripheral arteries. Eur Vasc Endovasc Surg. 2002; 24: 37-42.

8) Webster SE, Smith J, Thompson MM, Bell PR, Naylor AR. Does the sequence of clamp application during open abdominal aortic aneurysm surgery influence distal embolisation? Eur J Vasc Endovasc Surg. 2004; 27: 61-4.

9) Thompson MM, Smith JL, Bell PR. Thromboembolic complications during endovascular aneurysm repair. Semin Vasc Surg. 1999; 12: 215-9.

10) Görich J, Krämer S, Tomczak R, Seifarth H, Merkle E, Sunder-Plassmann L, et al. Thombo-embolic complications after endovascular aortic aneurysm repair. J Endovasc Ther. 2002; 9: 180-4. 